\title{
Finite-Time Stability and Stabilization of Itô-Type Stochastic Singular Systems
}

\author{
Zhiguo Yan ${ }^{1}$ and Weihai Zhang ${ }^{2}$ \\ ${ }^{1}$ School of Electrical Engineering and Automation, Qilu University of Technology, Jinan 250353, China \\ ${ }^{2}$ College of Information and Electrical Engineering, Shandong University of Science and Technology, Qingdao 266510, China \\ Correspondence should be addressed to Zhiguo Yan; yanzg500@sina.com
}

Received 14 October 2013; Accepted 19 November 2013; Published 14 January 2014

Academic Editor: Hui Zhang

Copyright (c) 2014 Z. Yan and W. Zhang. This is an open access article distributed under the Creative Commons Attribution License, which permits unrestricted use, distribution, and reproduction in any medium, provided the original work is properly cited.

\begin{abstract}
This paper is concerned with the finite-time stability and stabilization problems for linear Itô stochastic singular systems. The condition of existence and uniqueness of solution to such class of systems are first given. Then the concept of finite-time stochastic stability is introduced, and a sufficient condition under which an Itô stochastic singular system is finite-time stochastic stable is derived. Moreover, the finite-time stabilization is investigated, and a sufficient condition for the existence of state feedback controller is presented in terms of matrix inequalities. In the sequel, an algorithm is given for solving the matrix inequalities arising from finite-time stochastic stability (stabilization). Finally, two examples are employed to illustrate our results.
\end{abstract}

\section{Introduction}

Stochastic systems, especially for the systems governed by Itô-type stochastic differential equations, have received considerable attention due to its both theoretical and practical importance. Some results for this class of systems have been reported in the monographs and literatures, for example, stochastic stability and stabilization [1-3], linear/nonlinear stochastic $H_{\infty}$ control and filtering [4-7], and output tracking control for high-order stochastic nonlinear systems [8]. Meanwhile, singular systems (descriptor systems, implicit systems, generalized state-space systems, and differential-algebraic systems) have also attracted much attention of researchers and made a rapid progress. Many results have been achieved on different subjects related to such class of systems, for example, stability and impulsive elimination $[9,10]$, linear quadratic optimal control [11], and $H_{\infty}$ control/filtering and $H_{2} / H_{\infty}$ control [12-14]. Consequently, Itô stochastic singular systems have received attention in recent years. Reference [15] is concerned with the problems of stability of Itô singular stochastic systems with Markovian jumping. Reference [16] investigated the $H_{\infty}$ control/filtering for a class of singular stochastic time-delay systems. To the best of our knowledge, most of the results on stability of Itô stochastic singular systems are concerned with Lyapunov asymptotic stability or exponential stability, which is defined over an infinite-time interval.

In many practical situations, however, we are interested in stability of the system over a fixed finite-time interval. Such kind of stability is called finite-time stability (FTS). The concept of FTS was first introduced in the Russian literature. Later, this concept appeared in the western control literatures. Roughly speaking, a system is said to be finitetime stable if, given a bound on the initial condition, its state does not exceed a certain threshold during a specified time interval. Compared with infinite-time stability, the FTS can be used in the problem of controlling the trajectory of a space vehicle from an initial point to a final point in a prescribed time interval and all those applications where large values of the states should be attained, for instance, in the presence of saturations. Much effort has been devoted to FTS for its stability analysis and stabilization, for instance, linear continuous-time systems [17], linear discrete-time systems [18], stochastic systems [19-22], singular systems/Markovian jumping singular systems [23, 24], and stochastic singular biological economic systems [25]. Nevertheless, the FTS in [17-25] only requires that the state trajectory does not exceed a given upper bound during a prespecified time interval. 
Recently, [26] gave a new "finite-time stochastic stability" for linear Itô stochastic systems, which quantifies the state trajectory of some complex practical systems over a finitetime interval in more detail. Roughly speaking, a stochastic Itô system is called finite-time stochastically stable if its state trajectories do not exceed an upper bound $c_{2}$ and are not less than a lower bound $c_{1}\left(c_{1}<c_{2}\right)$ in the mean square sense during a specific time interval.

In this paper, motivated by [26], we consider finite-time stability and stabilization problems for Itô stochastic singular systems. Because of the special structure of Itô stochastic singular systems, the problems considered are of more complexity than those in [26]. By using stochastic analysis technology, the stability criterion and some stabilizing conditions are obtained. The contributions of this paper lie in the following three aspects: (1) the condition for the existence and uniqueness of solution to linear Itô stochastic singular systems is given. Our proof is different from that in [15], which may better reflect the essential characteristics of this class of systems. (2) The definition of finite-time stochastic stability for linear Itô stochastic singular systems is given. By the generalized Itô formula and mathematical expectation properties, some new stability criteria and the conditions of existence for state feedback controller are obtained as well. (3) A solving algorithm for the matrix inequalities arising from finite-time stochastic stability (stabilization) is given. By adjusting the parameters in this algorithm, the less conservative results can be attained.

The remainder of this paper is organized as follows. The definition of finite-time stochastic stability of linear Itô stochastic singular systems and some preliminaries are presented in Section 2. A sufficient condition to verify finitetime stochastic stability is given in Section 3. Section 4 gives some sufficient conditions for finite-time stochastic stabilization and a solving algorithm for the matrix inequalities arising from finite-time stochastic stability (stabilization). Section 5 employs two examples to illustrate the results. Finally, concluding remarks are made in Section 6.

Notation. $A^{T}$ is transpose of a matrix or vector $A . A>0$ $(A \geq 0)$ is positive definite (positive semidefinite) symmetric matrix. $I_{n \times n}$ is identity matrix. $\operatorname{tr}(A)$ is trace of a matrix $A . \lambda_{\max }(A)\left(\lambda_{\min }(A)\right)$ is the maximum (minimum) eigenvalue of a real symmetric matrix $A .\left(\Omega, \mathscr{F},\left\{\mathscr{F}_{t}\right\}_{t \geq 0}, \mathbb{P}\right)$ is a probability space with natural filtration $\left\{\mathscr{F}_{t}\right\}_{t \geq 0}$, and $\mathbb{E}[\cdot]$ stands for the mathematical expectation operator with respect to the given probability measure: $\delta(t)=\left\{\begin{array}{cc}\infty, & t=0, \\ 0, & \text { otherwise. }\end{array}\right.$

\section{Preliminaries and Problem Statement}

Consider an $n$-dimensional Itô stochastic singular system

$$
\begin{gathered}
E d x(t)=A x(t) d t+H x(t) d w(t), \\
x(0)=x_{0},
\end{gathered}
$$

on $t \geq 0$ with initial data $x(0)=x_{0} \in \mathbf{R}^{n} ; x(t) \in \mathbf{R}^{n}$ is the state vector; $E, A, H$ are $n \times n$-dimensional matrices with $0<\operatorname{rank}(E)=r \leq n . w(t)$ is a scalar Brownian motion defined on the probability space $\left(\Omega, \mathscr{F},\left\{\mathscr{F}_{t}\right\}_{t \geq 0}\right)$. In order to guarantee the existence and uniqueness of solution for the system (1), the following lemma is given.

Lemma 1. If there is a pair of nonsingular matrices $P \in$ $\mathbf{R}^{n \times n}, Q \in \mathbf{R}^{n \times n}$ or $P_{1} \in \mathbf{R}^{n \times n}, Q_{1} \in \mathbf{R}^{n \times n}$ for the triplet $(E, A, H)$ such that (at least) one of the following conditions is satisfied:

$$
\begin{gathered}
P E Q=\left[\begin{array}{cc}
I_{r} & 0 \\
0 & N_{n-r}
\end{array}\right], \\
\text { (I) } P A Q=\left[\begin{array}{cc}
A_{1} & 0 \\
0 & I_{n-r}
\end{array}\right], \\
P H Q=\left[\begin{array}{cc}
H_{1} & H_{2} \\
0 & 0_{n-r}
\end{array}\right], \\
P_{1} E Q_{1}=\left[\begin{array}{cc}
I_{r} & 0 \\
0 & 0_{n-r}
\end{array}\right], \\
P_{1} A Q_{1}=\left[\begin{array}{cc}
A_{11} & A_{12} \\
A_{21} & {\left[A_{22}\right]_{n-r}}
\end{array}\right], \\
P_{1} H Q_{1}=\left[\begin{array}{cc}
\mathscr{H}_{1} & \mathscr{H}_{2} \\
0 & 0_{n-r}
\end{array}\right],
\end{gathered}
$$

where $N$ is nilpotent matrix with nilpotent index $h$, then (1) has a unique solution.

Proof. Let $\zeta(t)=Q^{-1} x(t)=\left[\begin{array}{ll}\zeta_{1}^{T}(t) & \zeta_{2}^{T}(t)\end{array}\right]^{T}, \zeta_{1}^{T}(t) \in \mathbf{R}^{r}$ and $\zeta_{2}^{T}(t) \in \mathbf{R}^{n-r}$, then under the conditions of (I), the system (1) is equivalent to

$$
\begin{gathered}
d \zeta_{1}(t)=A_{1} \zeta_{1}(t) d t+\left[H_{1} \zeta_{1}(t)+H_{2} \zeta_{2}(t)\right] d w(t), \\
N d \zeta_{2}(t)=\zeta_{2}(t) d t
\end{gathered}
$$

which are called the slow and fast subsystems, respectively.

Note that the slow subsystem (4) is nothing more than an Itô stochastic differential equation. Applying the existence and uniqueness theorem of stochastic differential equations [27], the solution of (4) exists and is unique.

We note that the fast subsystem (5) is actually an ordinary differential equation. Taking the Laplace transforms on both sides of (5) and letting $\ell\left[\zeta_{2}(t)\right]=\Gamma(s)$, we have

$$
(s N-I) \Gamma(s)=N \zeta_{2}(0) .
$$

From this equation, we obtain

$$
\Gamma(s)=(s N-I)^{-1} N \zeta_{2}(0) .
$$

Taking the inverse Laplace transform on both sides of (7) gives

$$
\zeta_{2}(t)=-\sum_{i=1}^{h-1} \delta^{(i-1)}(t) N^{i} \zeta_{2}(0),
$$

which implies that (5) has a unique solution. So (1) has a unique solution. 
When triplet $(E, A, H)$ satisfies the condition (II), the proof can be referred to [15].

The proof is different from that in [15], which may better reflect the essential characteristics of this class of systems, such as, impulse behaviors. It is obviously observed from the proof of Lemma 1 that the response of system (1) may contain impulse terms. For convenience, we introduce the following definition.

Definition 2. If the state response of an Itô stochastic singular system, starting from an arbitrary initial value, does not contain impulse terms, then the system is called impulse-free.

Referring to some results on impulse-free of singular systems in [28], the following result is obtained.

Proposition 3. The following statements are equivalent under the conditions of Lemma 1:

(a) system (1) is impulse-free;

(b) $N=0$ in (2);

(c) $\operatorname{deg} \operatorname{det}(s E-A)=\operatorname{rank}(E)$;

(d) $\operatorname{det}\left(A_{22}\right) \neq 0$ in (3).

Proof. According to Definition 2 and the proof of Lemma 1, we can obtain the conclusion (a) $\Leftrightarrow$ (b).

By (2), $P(s E-A) Q=\operatorname{diag}\left(s I-A_{1}, s N-\right.$ I). Obviously, $N=0$ if and only if $\operatorname{deg} \operatorname{det}(s E-A)=$ $\operatorname{deg} \operatorname{det}\left(s I-A_{1}\right)=\operatorname{rank} E$. So, we get (b) $\Leftrightarrow$ (c).

By (3), it is easy to obtain that $\operatorname{det}\left(A_{22}\right) \neq 0$ if and only if $\operatorname{deg} \operatorname{det}(s E-A)=\operatorname{deg} \operatorname{det}\left[\begin{array}{cc}s I-A_{11} & -A_{12} \\ -A_{21} & -A_{22}\end{array}\right]=\operatorname{rank} E$. So, (c) $\Leftrightarrow(\mathrm{d})$.

Next, we extend the finite-time stochastic stability in [26] to Itô stochastic singular systems.

Definition 4. Given some positive scalars $c_{1}, c_{2}, c_{3}, c_{4}$, $T$ with $0<c_{1}<c_{3}<c_{4}<c_{2}$ and a positive definite matrix $R$, system (1) is said to be finite-time stochastically stable with respect to $\left(c_{1}, c_{2}, c_{3}, c_{4}, T, R\right)$, if

$$
\begin{aligned}
c_{3} & \leq x^{T}(0) E^{T} R E x(0) \leq c_{4} \\
& \Longrightarrow c_{1}<\mathbb{E}\left[x^{T}(t) E^{T} R E x(t)\right]<c_{2}, \quad \forall t \in[0, T] .
\end{aligned}
$$

Definition 4 can be described as follows: system (1) is said to be finite-time stochastically stable if, given a bound on the initial condition and a fixed time interval, its state trajectories are required to remain in a certain domain of ellipsoidal shape in the mean square sense during this time interval.

A 2-dimension case of Definition 4 is illustrated by Figure 1. A point $A$ lies in the shaped area. The trajectory starting from point $A$ cannot escape the disc from 0 to $c_{2}$ during the time interval $[0, T]$ in the mean square sense.

Remark 5. In [17-25], the finite-time stability only requires the state trajectory not to exceed a given upper bound. A 2dimension case of this finite-time stability can be illustrated by Figure 2. Nevertheless, the current finite-time stability

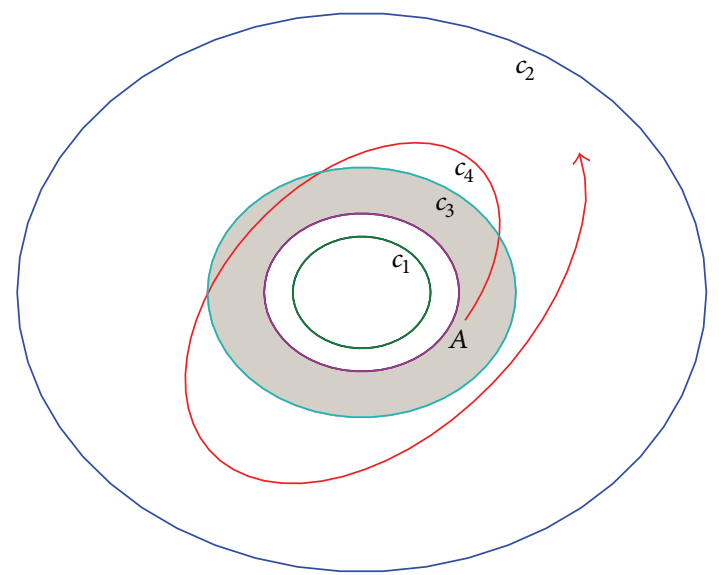

FIGURE 1: Illustration of finite-time stochastic stability in this paper.

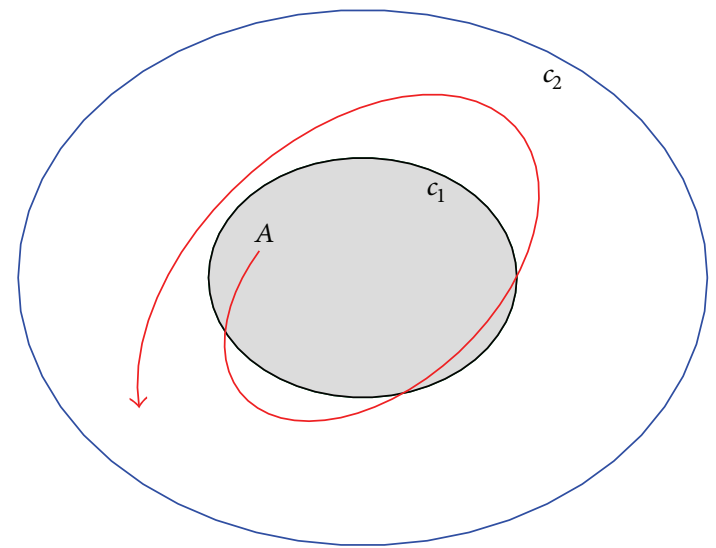

FIGURE 2: Illustration of finite-time stability in [17-25].

requires the state trajectory not only not to exceed a given upper bound but also not to be less than a given lower bound.

In the following, we give a proposition equivalent to Definition 4.

Proposition 6. System (1) is finite-time stochastically stable with respect to $\left(c_{1}, c_{2}, c_{3}, c_{4}, T, R\right)$ if and only if

$$
c_{3} \leq x^{T}(0) E^{T} R E x(0) \leq c_{4} \Longrightarrow c_{1}<\operatorname{tr}\left(E^{T} R E X(t)\right)<c_{2},
$$$$
\forall t \in(0, T] \text {, }
$$

where $X(t)>0$ is the solution to

$$
\begin{gathered}
E E^{T} \dot{X}(t)=A X(t) E^{T}+E X(t) A^{T}+H X(t) H^{T}, \\
X(0)=x(0) x^{T}(0) .
\end{gathered}
$$


Proof. Letting $X(t)=\mathbb{E}\left[x(t) x(t)^{T}\right]$, we easily obtain

$$
\begin{aligned}
\mathbb{E} & {\left[x^{T}(t) E^{T} R E x(t)\right] } \\
& =\mathbb{E} \operatorname{tr}\left(E^{T} R E x(t) x^{T}(t)\right)=\operatorname{tr}\left(E^{T} R E \mathbb{E} x(t) x^{T}(t)\right) \\
& =\operatorname{tr}\left(E^{T} R E X(t)\right) .
\end{aligned}
$$

Applying Itô's formula to $\mathbb{E}\left[x(t) E E^{T} x^{T}(t)\right]$, we obtain

$$
\begin{gathered}
E E^{T} \dot{X}(t)=A X(t) E^{T}+E X(t) A^{T}+H X(t) H^{T}, \\
X(0)=x(0) x^{T}(0) .
\end{gathered}
$$

Under the conditions of Lemma 1, (14) has unique solution $X(t)$. So the proof is completed.

By Kronecker's product theory, (14) can be rewritten as

$$
(E \otimes E) \dot{\vec{X}}(t)=[E \otimes A+A \otimes E+H \otimes H] \vec{X}(t),
$$

where $\vec{X}$ denotes the vector formed by stacking the rows of $X=\left(X_{i j}\right)_{n \times n}$ into one long vector; that is,

$$
\begin{gathered}
\vec{X}=\left[X_{11}, X_{12}, \ldots, X_{1 n}, X_{21}, X_{22}, \ldots,\right. \\
\left.X_{2 n}, \ldots, X_{n 1}, X_{n 2}, \ldots, X_{n n}\right]^{T},
\end{gathered}
$$

and $\otimes$ represents the Kronecker product of two matrices.

Remark 7. Proposition 6 is actually to solve a set of ordinary differential equations and avoids solving a stochastic differential equation (1), which provides an easier method to test finite-time stochastic stability of system (1).

Remark 8. If $E=I$, then system (1) becomes normal Itô stochastic systems and Proposition 6 reduces to Proposition 1 in [26].

Based on Definition 4, we define the finite-time stochastic stabilization as follows.

Definition 9. The following Itô stochastic singular controlled system

$$
E d x(t)=[A x(t)+B u(t)] d t+H x(t) d w(t)
$$

is said to be finite-time stochastically stabilizable, if there exists a state feedback control law $u(t)=K x(t)$, such that

$$
E d x(t)=(A+B K) x(t) d t+H x(t) d w(t)
$$

is finite-time stochastically stable.

Before proceeding further, we give some lemmas which will be used in the next sections.

Lemma 10 (Gronwall inequality). Letting $\theta(t)$ be a nonnegative function such that

$$
\theta(t) \leq a+b \int_{0}^{t} \theta(s) d s, \quad 0 \leq t \leq T
$$

for some constants $a, b \geq 0$, then one has

$$
\theta(t) \leq a \exp (b t), \quad 0 \leq t \leq T .
$$

Lemma 11 (modified Gronwall inequality [26]). Letting $\theta(t)$ be a nonnegative function such that

$$
\theta(t) \geq a+b \int_{0}^{t} \theta(s) d s, \quad 0 \leq t \leq T
$$

for some constants $a, b \geq 0$, then one has

$$
\theta(t) \geq a \exp (b t), \quad 0 \leq t \leq T .
$$

Lemma 12 (see [24]). (i) Assume that $\operatorname{rank}(E)=r$, there exist two nonsingular matrices $U$ and $V$ such that $E$ has the decomposition as

$$
\bar{E}=U E V=\operatorname{diag}\left\{I_{r}, 0\right\} .
$$

(ii) Define $\Phi=V\left[\begin{array}{ll}0 & I_{n-r}\end{array}\right]^{T}$, obviously, $\operatorname{rank} \Phi=n-$ $r, E \Phi=0$. If $P$ satisfies

$$
E P^{T}=P E^{T} \geq 0
$$

then $\widetilde{P}=U P\left[V^{-1}\right]^{T}$ with $U$ and $V$ satisfying (24) if and only if

$$
\widetilde{P}=\left[\begin{array}{cc}
P_{11} & P_{12} \\
0 & P_{22}
\end{array}\right]
$$

with $P_{11} \geq 0 \in \mathbf{R}^{r \times r}$. In addition, when $P$ is nonsingular, one has $P_{11}>0$ and $\operatorname{det}\left(P_{22}\right) \neq 0$. Furthermore, $P$ satisfying (25) can be parameterized as

$$
P=E V M V^{T}+U^{-1} N \Phi^{T}
$$

where $M=\operatorname{diag}\left\{P_{11}, * 1\right\}, N=\left[P_{12}^{T} P_{22}^{T}\right]^{T}$, and $\Phi \in$ $\mathbf{R}^{(n-r) \times(n-r)}$ is an arbitrary parameter matrix.

(iii) If $P$ is a nonsingular matrix, $R$ and $\Phi$ are two symmetric positive definite matrices, $P$ and $E$ satisfy (25), $M$ is a diagonal matrix from (27), and the following equality holds:

$$
P^{-1} E=E^{T} R^{1 / 2} Q R^{1 / 2} E .
$$

Then the symmetric positive definite matrix $Q=$ $R^{-1 / 2} U^{T} M^{-1} U R^{-1 / 2}$ is a solution of (28).

\section{Finite-Time Stochastic Stability}

In this section, we provide an impulse-free and finite-time stochastic stability condition for system (1). 
Theorem 13. Under the conditions of Lemma 1, if there exist positive matrices $Q>0$, nonsingular matrix $P$, and two scalar $\alpha \geq 0$ and $\beta \geq 0$ satisfying

$$
\begin{gathered}
E^{T} P=P^{T} E \geq 0 \\
E^{T} P=E^{T} R^{1 / 2} Q R^{1 / 2} E \\
\lambda_{\max }(Q) c_{4} e^{\alpha T}-c_{2} \lambda_{\min }(Q)<0 \\
c_{1} \lambda_{\max }(Q)-c_{3} \lambda_{\min }(Q)<0 \\
A^{T} P+P^{T} A+H^{T} \widetilde{Q} H-\alpha E^{T} P<0 \\
\beta E^{T} P-A^{T} P-P^{T} A+H^{T} \widetilde{Q} H<0,
\end{gathered}
$$

then the system (1) is impulse-free and finite-time stochastically stable with respect to $\left(c_{1}, c_{2}, c_{3}, c_{4}, T, R\right)$.

Proof. We split the proof of Theorem 13 into three steps as follows.

Step 1. We prove system (1) to be impulse-free. By condition (33), we obtain

$$
P^{T} A+A^{T} P-\alpha E^{T} P<0 .
$$

Take nonsingular $U$ and $V$ such that $E$ has the decomposition as

$$
\bar{E}=U E V=\operatorname{diag}\left\{I_{r}, 0\right\}
$$

Denote

$$
\begin{gathered}
\bar{A}=U A V=\left[\begin{array}{ll}
A_{11} & A_{12} \\
A_{21} & A_{22}
\end{array}\right], \\
\bar{P}=\left[U^{-1}\right]^{T} P V=\left[\begin{array}{ll}
P_{11} & P_{12} \\
P_{21} & P_{22}
\end{array}\right] .
\end{gathered}
$$

From (36), (37), and (29), it is easy to obtain

$$
P_{11} \geq 0, \quad P_{12}=0 \text {. }
$$

Substitute (37) and (38) into (35), then it becomes

$$
\left[\begin{array}{cc}
\Sigma_{1} & \Sigma_{2} \\
\Sigma_{2}^{T} & P_{22}^{T} A_{22}+A_{22}^{T} P_{22}
\end{array}\right]<0,
$$

where $\Sigma_{1}=P_{11}^{T} A_{11}+P_{21}^{T} A_{21}+A_{11}^{T} P_{11}+A_{21}^{T} P_{21}-\alpha P_{11}, \Sigma_{2}=$ $P_{11}^{T} A_{12}+P_{21}^{T} A_{22}+A_{21}^{T} P_{22}$.

From (39), $A_{22}^{T} P_{22}+P_{22}^{T} A_{22}<0$, which implies that $A_{22}$ is nonsingular, by Proposition 3, system (1) is impulse-free.

Step 2. $x^{T}(0) E^{T} R E x(0)<c_{4} \Rightarrow \mathbb{E}\left[x^{T}(t) E^{T} R E x(t)\right]<c_{2}$.

Construct a stochastic quadratic function as

$$
V(x(t))=x^{T}(t) E^{T} P x(t),
$$

where $P$ satisfies (29)-(33).
Applying generalized Itô formula $[1,15]$ for $V(x(t))$ along the trajectory of system (1) and considering condition (29), we have

$$
\begin{gathered}
\mathscr{L} V(x(t))=[A x(t)]^{T} P x(t)+x^{T}(t) P^{T}[A x(t)] \\
+[H x(t)]^{T} \widetilde{Q}[H x(t)]
\end{gathered}
$$

which leads to

$$
\begin{aligned}
& \mathscr{L} V(x(t))-\alpha V(x(t)) \\
& \quad=x^{T}(t)\left[A^{T} P+P^{T} A+H^{T} \widetilde{Q} H-\alpha E^{T} P\right] x(t) .
\end{aligned}
$$

By condition (33), it is easy to see that

$$
\mathscr{L} V(x(t))<\alpha V(x(t)) .
$$

Integrating both sides of (43) from 0 to $t$ with $t \in$ $[0, T]$ and then taking the expectation, it yields that

$$
\mathbb{E} V(x(t))<V(x(0))+\alpha \int_{0}^{t} \mathbb{E} V(x(s)) d s .
$$

By Lemma 10, we obtain

$$
\mathbb{E} V(x(t))<V(x(0)) e^{\alpha t} .
$$

According to condition (30), it follows that

$$
\begin{aligned}
\mathbb{E} V(x(t)) & =\mathbb{E}\left[x^{T}(t) E^{T} P x(t)\right] \\
& =\mathbb{E}\left[x^{T}(t) E^{T} R^{1 / 2} Q R^{1 / 2} E x(t)\right] \\
& \geq \lambda_{\min }(Q) \mathbb{E}\left[x^{T}(t) E^{T} R E x(t)\right], \\
V(x(0)) e^{\alpha t} & =\left[x^{T}(0) E^{T} P E x(0)\right] e^{\alpha t} \\
& =\left[x^{T}(0) E^{T} R^{1 / 2} Q R^{1 / 2} E x(0)\right] e^{\alpha t} \\
& \leq\left[\lambda_{\max }(Q) x^{T}(0) E^{T} R E x(0)\right] e^{\alpha t} \\
& \leq\left[\lambda_{\max }(Q)\right] c_{1} e^{\alpha T} .
\end{aligned}
$$

From (46), we easily obtain

$$
\mathbb{E}\left[x^{T}(t) E^{T} R E x(t)\right] \leq \frac{\lambda_{\max }(Q)}{\lambda_{\min }(Q)} c_{1} e^{\alpha T} .
$$

By condition (31), it is obvious that $\mathbb{E}\left[x^{T}(t) E^{T} R E x(t)\right]<$ $c_{2}$

Step 3. $c_{3}<x^{T}(0) E^{T} R E x(0) \Rightarrow c_{1}<\mathbb{E}\left[x^{T}(t) E^{T} R E x(t)\right]$.

By (34) and (41), we obtain

$$
\mathscr{L} V(x(t))>\beta V(x(t)) .
$$

Integrating both sides of (48) from 0 to $t$ with $t \in$ $[0, T]$ and then taking the expectation, it yields that

$$
\mathbb{E} V(x(t))>V(x(0))+\beta \int_{0}^{t} \mathbb{E} V(x(s)) d s .
$$


By Lemma 11, we conclude that

$$
\mathbb{E} V(x(t))>V(x(0)) e^{\beta t}
$$

According to condition (30), it follows that

$$
\begin{aligned}
\mathbb{E} V(x(t))= & \mathbb{E}\left[x^{T}(t) E^{T} P x(t)\right] \\
= & \mathbb{E}\left[x^{T}(t) E^{T} R^{1 / 2} Q R^{1 / 2} E x(t)\right] \\
\leq & \lambda_{\max }(Q) \mathbb{E}\left[x^{T}(t) E^{T} R E x(t)\right], \\
V(x(0)) e^{\beta t} & =\left[x^{T}(0) E^{T} P x(0)\right] e^{\beta t} \\
& =\left[x^{T}(0) E^{T} R^{1 / 2} Q_{1} R^{1 / 2} E x(0)\right] e^{\beta t} \\
& \geq\left[\lambda_{\min }(Q) x^{T}(0) E^{T} R E x(0)\right] e^{\beta t} \\
& \geq\left[\lambda_{\min }(Q)\right] c_{3} .
\end{aligned}
$$

From (32), (51), we obtain

$$
c_{1}<\frac{c_{3} \lambda_{\min }(Q)}{\lambda_{\max }(Q)}<\mathbb{E}\left[x^{T}(t) E^{T} R E x(t)\right] .
$$

So, the proof is completed.

Theorem 13 provides a criterion for finite-time stochastic stability of system (1). To design finite-time controller conveniently, the following corollary is given.

Corollary 14. Under the conditions of Lemma 1, if there exist positive matrices $Q>0$, nonsingular matrix $\bar{P}$, and two scalars $\alpha \geq 0, \beta \geq 0$ satisfying

$$
\begin{gathered}
E \bar{P}^{T}=\bar{P} E^{T} \geq 0 \\
\bar{P}^{-1} E=E^{T} R^{1 / 2} Q R^{1 / 2} E \\
\lambda_{\max }(Q) c_{4} e^{\alpha T}-c_{2} \lambda_{\min }(Q)<0 \\
c_{1} \lambda_{\max }(Q)-c_{3} \lambda_{\min }(Q)<0 \\
\bar{P} A^{T}+A \bar{P}^{T}+\bar{P} H^{T} \widetilde{Q} H \bar{P}^{T}-\alpha \bar{P} E^{T}<0 \\
\beta \bar{P} E^{T}-\bar{P} A^{T}-A \bar{P}^{T}+\bar{P} H^{T} \widetilde{Q} H \bar{P}^{T}<0,
\end{gathered}
$$

then the system (1) is impulse-free and finite-time stochastically stable with respect to $\left(c_{1}, c_{2}, c_{3}, c_{4}, T, R\right)$.

Proof. Premultiply and postmultiply (53) by the matrices $\bar{P}^{-1}$ and $\left[\bar{P}^{-1}\right]^{T}$. Premultiply and postmultiply (57) and (58) by $\bar{P}^{-1}$ and $\left[\bar{P}^{-1}\right]^{T}$. Let $\left[\bar{P}^{-1}\right]^{T}=P$, by $(29)-(33)$, this proof completes.

Remark 15. If $E=I$, then Corollary 14 reduces to Theorem 1 in [26].

\section{Finite-Time Stochastic Stabilization}

In this section, we aim to design a finite-time stabilizing controller for system (18). To this aim, the following result is obtained.

Theorem 16. Under the conditions of Lemma 1, if there exist positive matrices $Q>0$, nonsingular matrix $\bar{P}$, and two scalars $\alpha \geq 0, \beta \geq 0$ satisfying (53)-(56) and matrix inequalities

$$
\begin{aligned}
& \bar{P} A^{T}+X^{T} B^{T}+A \bar{P}^{T}+B X+\bar{P} H^{T} \widetilde{Q} H \bar{P}^{T}-\alpha \bar{P} E^{T}<0 \\
& \beta \bar{P} E^{T}-\bar{P} A^{T}-X^{T} B^{T}-A \bar{P}^{T}-B X+\bar{P} H^{T} \widetilde{Q} H \bar{P}^{T}<0
\end{aligned}
$$

then system (18) is impulse-free and finite-time stochastically stabilizable with respect to $\left(c_{1}, c_{2}, c_{3}, c_{4}, T, R\right)$. In addition, the feedback controller gain can then be given by $K=X\left[\bar{P}^{-1}\right]^{T}$.

Proof. If the state feedback controller is taken into account, then the state equation of system (18) becomes

$$
E d x(t)=[(A+B K) x(t)] d t+H x(t) d w(t), \quad t \in[0, T] .
$$

Therefore, we can replace $A$ by $A+B K$ in Corollary 14. As a result, condition (57) and (58) turn to

$$
\bar{P} A^{T}+\bar{P} K^{T} B^{T}+A \bar{P}^{T}+B K \bar{P}^{T}+\bar{P} H^{T} \widetilde{Q} H \bar{P}^{T}-\alpha \bar{P} E^{T}<0,
$$

$$
\beta \bar{P} E^{T}-\bar{P} A^{T}-\bar{P} K^{T} B^{T}-A \bar{P}^{T}-B K \bar{P}^{T}+\bar{P} H^{T} \widetilde{Q} H \bar{P}^{T}<0 .
$$

By setting $X=K \bar{P}^{T},(62)$ and (63) become (59) and (60), respectively. This completes the proof of Theorem 16 .

On the basis of Theorem 16, the following theorem gives a sufficient condition for designing a finite-time stabilizing controller of (18), which is easy to solve.

Theorem 17. Under the conditions of Lemma 1, if there exist positive matrices $M=\operatorname{diag}\left\{P_{11}, * 1\right\}>0, Q>0$, matrices $N \in \mathbf{R}^{n \times(n-r)}, X$, and scalars $\alpha \geq 0, \beta \geq 0, \lambda_{1}>$ $0, \lambda_{2}>0$, satisfying the following matrix inequalities:

$$
\begin{gathered}
{\left[\begin{array}{cc}
\Omega-\alpha \bar{P} E & \bar{P} H^{T} U^{T} \\
* & -M
\end{array}\right]<0} \\
{\left[\begin{array}{cc}
\beta \bar{P} E-\Omega & \bar{P} H^{T} U^{T} \\
* & -M
\end{array}\right]<0} \\
\lambda_{1} I<R^{1 / 2}\left[U^{-1}\right]^{\prime} M U^{-1} R^{1 / 2}<\lambda_{2} I \\
\lambda_{2} c_{4} e^{\alpha T}-c_{2} \lambda_{1}<0 \\
c_{1} \lambda_{2}-c_{3} \lambda_{1}<0,
\end{gathered}
$$


then there exists a controller such that close-loop system of system (18) is impulse-free and finite-time stochastically stable with respect to $\left(c_{1}, c_{2}, c_{3}, c_{4}, T, R\right)$, where $\Omega=\bar{P} A^{T}+X^{T} B^{T}+$ $A \bar{P}^{T}+B X, \bar{P}(M, N)=E V M V^{T}+U^{-1} N \Phi^{T}$ is nonsingular. In addition, the feedback controller is $u(t)=X\left[\bar{P}^{T}(M, N)\right]^{-1} x(t)$.

Proof. By Lemma 12, $\bar{P}$ satisfying (53) in Theorem 16 can be parameterized as

$$
\bar{P}=E V M V^{T}+U^{-1} N \Phi^{T}
$$

and (54) holds when $Q=R^{-1 / 2} U^{T} M^{-1} U R^{-1 / 2}$, where $M=$ $\operatorname{diag}\left\{P_{11}, * 1\right\}, N=\left[P_{12}^{T} P_{22}^{T}\right]^{T}$.

Substituting $Q=R^{-1 / 2} U^{T} M^{-1} U R^{-1 / 2}$ into (59) and (60), by Schur Complement, (53) and (65) are obtained, respectively.

Since

$$
\begin{aligned}
& \lambda_{\text {max }}(Q)=\left[\lambda_{\min }\left(R^{1 / 2}\left[U^{-1}\right]^{T} M U^{-1} R^{1 / 2}\right)\right]^{-1}, \\
& \lambda_{\min }(Q)=\left[\lambda_{\max }\left(R^{1 / 2}\left[U^{-1}\right]^{T} M U^{-1} R^{1 / 2}\right)\right]^{-1},
\end{aligned}
$$

it is easy to check that conditions (55)-(56) are guaranteed by (66)-(68). This completes the proof.

Remark 18. It is important to notice that once we have fixed the values for $\alpha$ and $\beta$, the feasibility of the conditions stated in Theorem 17 can be turned into the following LMIs based feasibility problem. The algorithm of how to choose $\alpha$ and $\beta$ for Theorem 17 is given in the following.

Algorithm 19. Consider the following steps.

Step 1. Given $c_{1}, c_{2}, c_{3}, c_{4}, R$, and $T$.

Step 2. Take a series of $\alpha_{i}(i=1, \ldots, n)$ by a step size $d_{1}$ and a series of $\beta_{j}(j=1, \ldots, m)$ by a step size $d_{2}$.

Step 3. Set $i=1$, take a $\alpha_{i}$.

Step 4. Set $j=1$, take a $\beta_{j}$.

Step 5. If $\left(\alpha_{i}, \beta_{j}\right)$ makes (53)-(68) have feasible solutions, then store $\left(\alpha_{i}, \beta_{j}\right)$ into $(X(i), Y(j))$ and $\beta_{j}=\beta_{j+1}$, go to Step 5; otherwise go to Step 6.

Step 6. If $i+1<n$, then $\alpha_{i}=\alpha_{i+1}$ and take $\beta_{1}$, go to Step 5 . Otherwise, go to Step 7.

Step 7. Stop. If $(X, Y)=(0,0)$, then we cannot find $(\alpha, \beta)$ making (53)-(68) have feasible solution; otherwise, there exists $(\alpha, \beta)$ making (53)-(68) have feasible solution.

Remark 20. By Algorithm 19, we can obtain a region surrounded by $\alpha$ and $\beta$, if it exists, which is used to select $\alpha$ and $\beta$ for appropriate conditions.
Remark 21. If $\bar{P}(M, N)$ obtained from LMIs (53)-(68) is singular, then we can adjust $\alpha$ and $\beta$ such that $\bar{P}(M, N)$ is non-singular.

\section{Examples}

In this section, we will present two examples to illustrate the obtained results.

Example 1. Consider the Itô stochastic singular system (1) with

$$
\begin{gathered}
E=\left[\begin{array}{ll}
1 & 0 \\
2 & 0
\end{array}\right], \quad A=\left[\begin{array}{cc}
-1 & -2 \\
-2 & 3
\end{array}\right], \quad H=\left[\begin{array}{ll}
1 & 1 \\
2 & 2
\end{array}\right], \\
x(0)=\left[\begin{array}{ll}
1.6 & 0
\end{array}\right]^{\prime}, \quad c_{1}=1, \quad c_{2}=20, \\
c_{3}=10, \quad c_{4}=15, \quad T=0.25, \quad R=I .
\end{gathered}
$$

For system (1), there exists a pair of nonsingular matrices

$$
U=\left[\begin{array}{rr}
-\frac{3}{2} & 1 \\
-2 & 1
\end{array}\right], \quad V=\left[\begin{array}{ll}
2 & 0 \\
0 & 2
\end{array}\right]
$$

such that

$$
U E V=\left[\begin{array}{ll}
1 & 0 \\
0 & 0
\end{array}\right], \quad U A V=\left[\begin{array}{cc}
-1 & 0 \\
0 & 2
\end{array}\right], \quad U H V=\left[\begin{array}{ll}
1 & 1 \\
0 & 0
\end{array}\right],
$$

which satisfy Lemma 1, so system (1) has a unique solution and is also impulse-free. We find that system (1) is equivalent to the following system:

$$
\begin{gathered}
d x_{1}(t)=-x_{1}(t) d t+x_{1}(t) d w(t), \\
x_{2}(t)=0 .
\end{gathered}
$$

Based on this, $\mathbb{E}\left[x^{T}(t) E^{T} R E x(t)\right]=\mathbb{E}\left[5 x_{1}^{2}(t)\right]$.

By Proposition 3, we solve (16), where

$$
E \otimes E=\left[\begin{array}{llll}
1 & 0 & 0 & 0 \\
2 & 0 & 0 & 0 \\
2 & 0 & 0 & 0 \\
4 & 0 & 0 & 0
\end{array}\right]
$$

$$
E \otimes A+A \otimes E+H \otimes H=\left[\begin{array}{cccc}
-1 & -1 & -1 & 1 \\
-2 & 5 & -2 & 2 \\
-2 & -2 & 5 & 2 \\
-4 & 10 & 10 & 4
\end{array}\right]
$$

By a simple calculation, we obtain $\vec{X}(t)=\left[\begin{array}{llll}2.56 e^{-t} & 0 & 0 & 0\end{array}\right]$. It is easy to obtain that $1<\operatorname{tr}\left(E^{T} R E X(t)\right)=5 \times 2.56 e^{-t}<$ $20, t \in[0,0.25]$, so (1) is finite-time stochastically stable with respect to $(1,20,10,15,0.25, I)$. Figure 3 depicts the evolution of $\mathbb{E}\left[x^{T}(t) E^{T} R E x(t)\right]$ of system (1). 


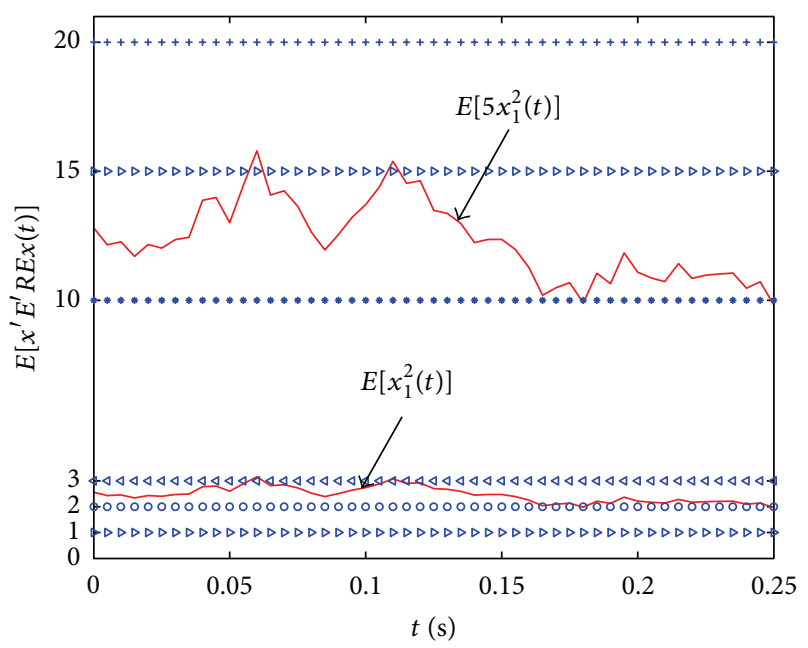

Figure 3: The evolution of $\mathbb{E}\left[x^{T}(t) E^{T} R E x(t)\right]$ of system (1).

Example 2. Consider the Itô stochastic singular system (18) with

$$
\begin{gathered}
E=\left[\begin{array}{lll}
1 & 0 & 0 \\
0 & 1 & 0 \\
0 & 0 & 0
\end{array}\right], \quad A=\left[\begin{array}{ccc}
0.8 & 1 & 0 \\
-0.2 & 1 & 0 \\
0 & 0 & 1
\end{array}\right], \\
H=\left[\begin{array}{lll}
1 & 1 & 2 \\
2 & 1 & 2 \\
0 & 0 & 0
\end{array}\right], \quad B=\left[\begin{array}{lll}
0.8 & 1 & 1
\end{array}\right]^{T}, \\
x_{1}(0)=1.5, \quad x_{2}(0)=1, \quad x_{3}(0)=0, \quad c_{1}=1, \\
c_{2}=20, \quad c_{3}=3, \quad c_{4}=4, \quad T=0.5, \quad R=I .
\end{gathered}
$$

By Lemma 12, we obtain $U=I, V=I$ and $\Phi=\left[\begin{array}{lll}0 & 0 & 1\end{array}\right]^{T}$. Apply Algorithm 19 to Theorem 17, a region surrounded by $\alpha$ and $\beta$, which is illustrated in Figure 4 .

Selecting $\alpha=2, \beta=0.2$ and solving (53)-(68), we obtain

$$
\begin{aligned}
& M=\left[\begin{array}{ccc}
98.8729 & 12.1890 & 0 \\
12.1890 & 137.9050 & 0 \\
0 & 0 & 114.8137
\end{array}\right], \\
& N=\left[\begin{array}{lll}
-71.1246 & -77.6051 & -7.9438
\end{array}\right]^{T}, \\
& X=\left[\begin{array}{lll}
-50.0746 & -76.8002 & 26.0568
\end{array}\right], \\
& \lambda_{1}=87.0062, \quad \lambda_{2}=154.6197 .
\end{aligned}
$$

Hence, the feedback gain matrix is given by

$$
K=X[E M+N \Phi]^{-T}=\left[\begin{array}{lll}
-2.5981 & -2.1731-3.2801
\end{array}\right]
$$

Under the following state feedback controller

$$
u(t)=\left[\begin{array}{lll}
-2.5981 & -2.1731 & -3.2801
\end{array}\right] x(t),
$$

the closed-loop system of (18) is impulse-free and finite-time stochastically stable with respect to $(1,20,3,4,0.5, I)$.

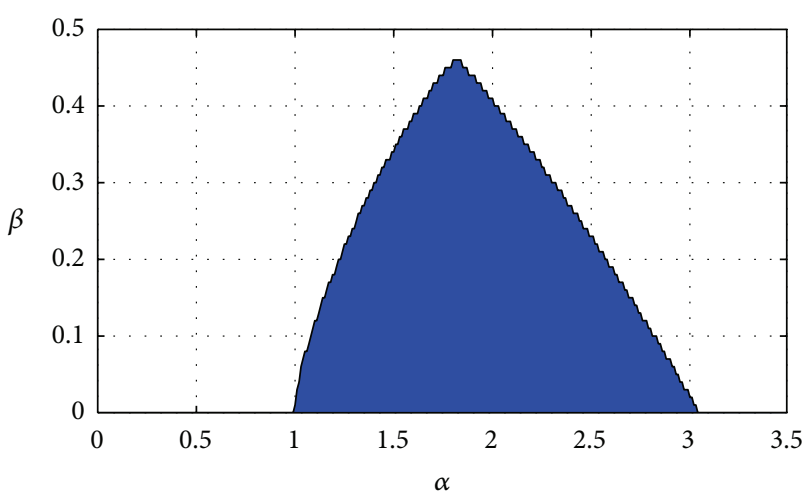

FIgURe 4: A region by $\alpha$ and $\beta$.

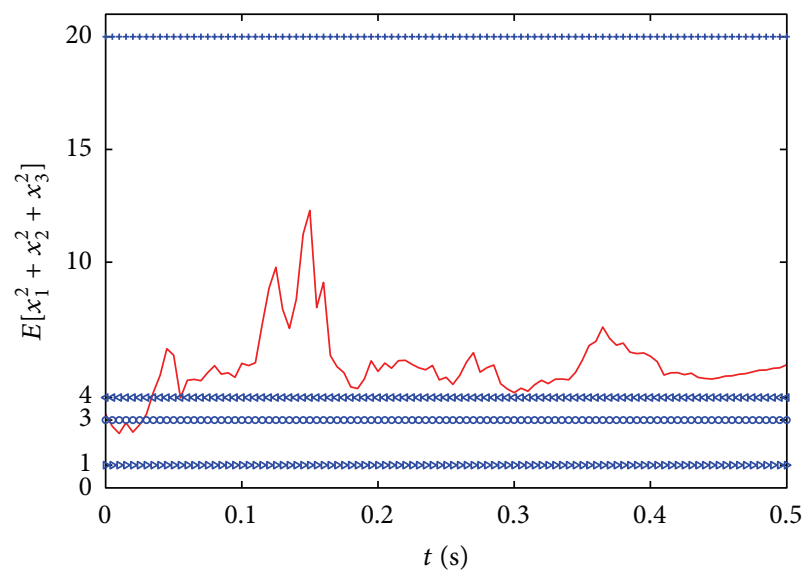

FIGURE 5: The evolution of $\mathbb{E}\left[x^{T}(t) E^{T} R E x(t)\right]$ of the closed-system of (18).

Figure 5 depicts the evolution of $\mathbb{E}\left[x^{T}(t) E^{T} \operatorname{RE} x(t)\right]$ of system (18). Figure 6 gives the evolution of $u(t)$.

\section{Conclusion}

In this paper, we have dealt with finite-time stability and stabilization problems for linear Itô stochastic singular systems and also established a condition of the existence and uniqueness of solution of linear Itô stochastic singular systems. A new sufficient condition has been provided to guarantee that the linear Itô stochastic singular system is impulse-free and finite-time stochastic stable. Based on the obtained result, we have also derived the corresponding stabilization criteria. Moreover, the finite-time stochastic stabilization has been studied via state feedback, and some new sufficient conditions have been given. Two examples are presented to illustrate the effectiveness of the proposed results. In addition, we can refer to [29-31] and extend the results of this paper to Markovian jump systems, networked systems, and linear parameter varying systems. 


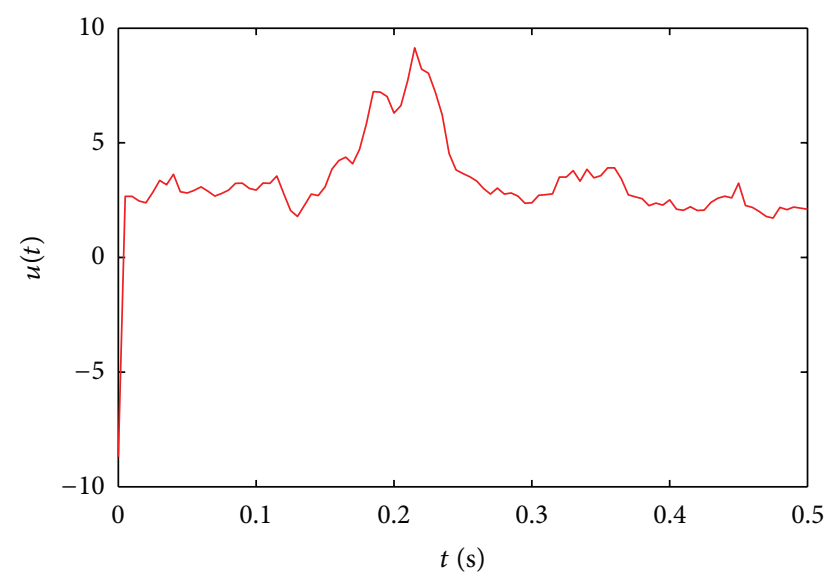

Figure 6: The evolution of $u(t)$.

\section{Conflict of Interests}

The authors declare that there is no conflict of interests regarding the publication of the paper.

\section{Acknowledgments}

This work is supported by the Starting Research Foundation of Qilu University of Technology (Grant no. 12045501), NSF of Shandong Province (Grant no. ZR2013FM022), NSF of China (Grant no. 61174078), the Research Fund for the Taishan Scholar Project of Shandong Province of China and the SDUST Research Fund (Grant no. 2011KYTD105), and the State Key Laboratory of Alternate Electrical Power System with Renewable Energy Sources (Grant no. LAPS13018).

\section{References}

[1] X. Mao, Stochastic Differential Equations and Applications, Horwood Publishing, London, UK, 2nd edition, 2008.

[2] W. Zhang and B.-S. Chen, " $\mathscr{H}$-representation and applications to generalized Lyapunov equations and linear stochastic systems," IEEE Transactions on Automatic Control, vol. 57, no. 12, pp. 3009-3022, 2012.

[3] Z. Lin, J. Liu, W. Zhang, and Y. Niu, "Stabilization of interconnected nonlinear stochastic Markovian jump systems via dissipativity approach," Automatica, vol. 47, no. 12, pp. 27962800, 2011.

[4] D. Hinrichsen and A. J. Pritchard, "Stochastic $H_{\infty}$," SIAM Journal on Control and Optimization, vol. 36, no. 5, pp. 1504-1538, 1998.

[5] W. Zhang and B.-S. Chen, "State feedback $H_{\infty}$ control for a class of nonlinear stochastic systems," SIAM Journal on Control and Optimization, vol. 44, no. 6, pp. 1973-1991, 2006.

[6] Z. Lin, Y. Lin, and W. Zhang, "A unified design for state and output feedback $H_{\infty}$ control of nonlinear stochastic Markovian jump systems with state and disturbance-dependent noise," Automatica, vol. 45, no. 12, pp. 2955-2962, 2009.

[7] E. Gershon, D. J. N. Limebeer, U. Shaked, and I. Yaesh, "Robust $H_{\infty}$ filtering of stationary continuous-time linear systems with stochastic uncertainties," IEEE Transactions on Automatic Control, vol. 46, no. 11, pp. 1788-1793, 2001.
[8] X.-J. Xie and N. Duan, "Output tracking of high-order stochastic nonlinear systems with application to benchmark mechanical system," IEEE Transactions on Automatic Control, vol. 55, no. 5, pp. 1197-1202, 2010.

[9] J. Y. Ishihara and M. H. Terra, "On the Lyapunov theorem for singular systems," IEEE Transactions on Automatic Control, vol. 47, no. 11, pp. 1926-1930, 2002.

[10] G. Zhang and W. Liu, "Impulsive mode elimination for descriptor systems by a structured P-D feedback," IEEE Transactions on Automatic Control, vol. 56, no. 12, pp. 2968-2973, 2011.

[11] J. Zhu, S. Ma, and Z. Cheng, "Singular LQ problem for nonregular descriptor systems," IEEE Transactions on Automatic Control, vol. 47, no. 7, pp. 1128-1133, 2002.

[12] S. Xu, J. Lam, and Y. Zou, " $H_{\infty}$ filtering for singular systems," IEEE Transactions on Automatic Control, vol. 48, no. 12, pp. 2217-2222, 2003.

[13] L. Zhang, B. Huang, and J. Lam, "LMI synthesis of $\mathrm{H}_{2}$ and mixed $\mathrm{H}_{2} / \mathrm{H}_{\mathrm{\infty}}$ controllers for singular systems," IEEE Transactions on Circuits and Systems II, vol. 50, no. 9, pp. 615-626, 2003.

[14] Z. Yan, G. Zhang, and J. Wang, "Infinite horizon $H_{2} / H_{\infty}$ control for descriptor systems: nash game approach," Journal of Control Theory and Applications, vol. 10, no. 2, pp. 159-165, 2012.

[15] L. Huang and X. Mao, "Stability of singular stochastic systems with Markovian switching," IEEE Transactions on Automatic Control, vol. 56, no. 2, pp. 424-429, 2011.

[16] J. Xia, Robust control and filter for continuous stochstic time-delay systems [Ph.D. dissertation], Nanjing University of Science and Technology, Nanjing, China, 2007.

[17] F. Amato, M. Ariola, and P. Dorato, "Finite-time control of linear systems subject to parametric uncertainties and disturbances," Automatica, vol. 37, no. 9, pp. 1459-1463, 2001.

[18] F. Amato and M. Ariola, "Finite-time control of discrete-time linear systems," IEEE Transactions on Automatic Control, vol. 50, no. 5, pp. 724-729, 2005.

[19] W. Zhang and X. An, "Finite-time control of linear stochastic systems," International Journal of Innovative Computing, Information and Control, vol. 4, no. 3, pp. 687-694, 2008.

[20] Z. Yan, G. Zhang, and J. Wang, "Non-fragile robust finitetime $H_{\infty}$ control for nonlinear stochastic Itô systems using neural network," International Journal of Control, Automation and Systems, vol. 10, no. 5, pp. 873-882, 2012.

[21] Z. G. Yan and G. S. Zhang, "Finite-time $H_{\infty}$ control for linear stochastic systems," Control and Decision, vol. 26, no. 8, pp. 1224-1228, 2011.

[22] Z. G. Yan and G. S. Zhang, "Finite-time $H_{\infty}$ filtering for a class of nonlinear stochastic uncertain systems," Control and Decision, vol. 29, no. 3, pp. 419-424, 2012.

[23] J. Feng, Z. Wu, and J. Sun, "Finite-time control of linear singular systems with parametric uncertainties and disturbances," Acta Automatica Sinica, vol. 31, no. 4, pp. 634-637, 2005.

[24] Y. Zhang, C. Liu, and X. Mu, "Robust finite-time $H_{\infty}$ control of singular stochastic systems via static output feedback," Applied Mathematics and Computation, vol. 218, no. 9, pp. 5629-5640, 2012.

[25] S. Xing, Q. Zhang, and Y. Zhang, "Finite-time stability analysis and control for a class of stochastic singular biological economic systems based on T-S fuzzy model," Abstract and Applied Analysis, vol. 2013, Article ID 946491, 10 pages, 2013.

[26] Z. Yan, G. Zhang, and W. Zhang, "Finite-time stability and stabilization of linear Itô stochastic systems with state and control-dependent noise," Asian Journal of Control, vol. 15, no. 1, pp. 270-281, 2013. 
[27] B. Oksendal, Stochastic Differential Equations: An Introduction with Applications, Springer, New York, NY, USA, 5th edition, 2000.

[28] L. Dai, Singular Control Systems, Springer, Berlin, Germany, 1989.

[29] H. Zhang, J. Wang, and Y. Shi, "Robust $H_{\infty}$ sliding-mode control for Markovian jump systems subject to intermittent observations and partially known transition probabilities," Systems \& Control Letters, vol. 62, no. 12, pp. 1114-1124, 2013.

[30] H. Zhang, Y. Shi, and M. Liu, " $H_{\infty}$ switched filtering for networked systems based on delay occurrence probabilities," Journal of Dynamic Systems, Measurement, and Control, vol. 135, no. 6, Article ID 061002, 5 pages, 2013.

[31] H. Zhang, Y. Shi, and A. S. Mehr, "Parameter-dependent mixed $\mathrm{H}_{2} / \mathrm{H}_{\mathrm{\infty}}$ filtering for linear parameter-varying systems," IET Signal Processing, vol. 6, no. 7, pp. 697-703, 2012. 


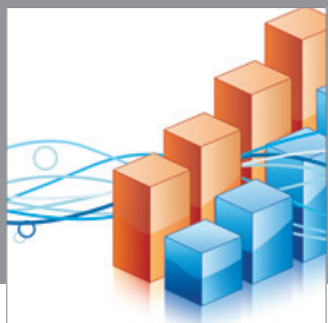

Advances in

Operations Research

mansans

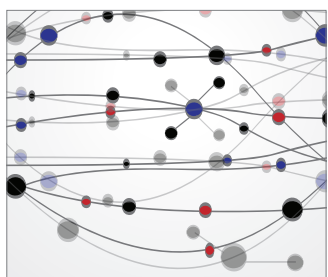

The Scientific World Journal
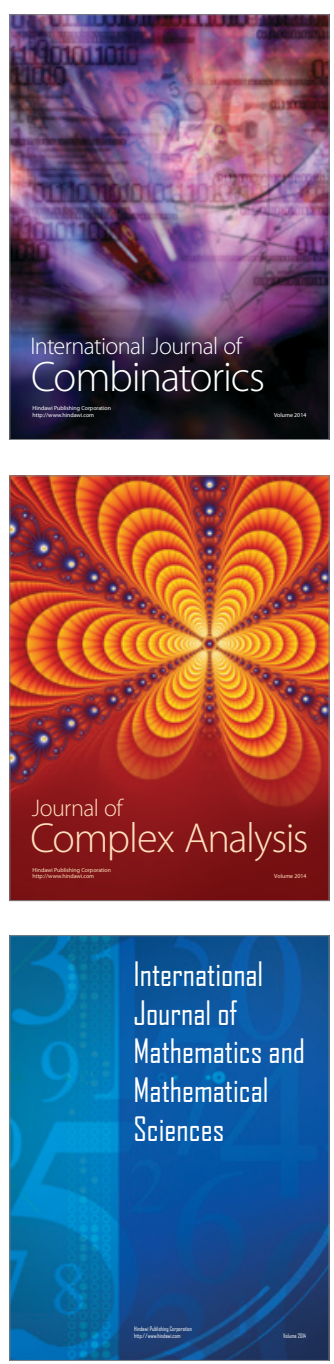
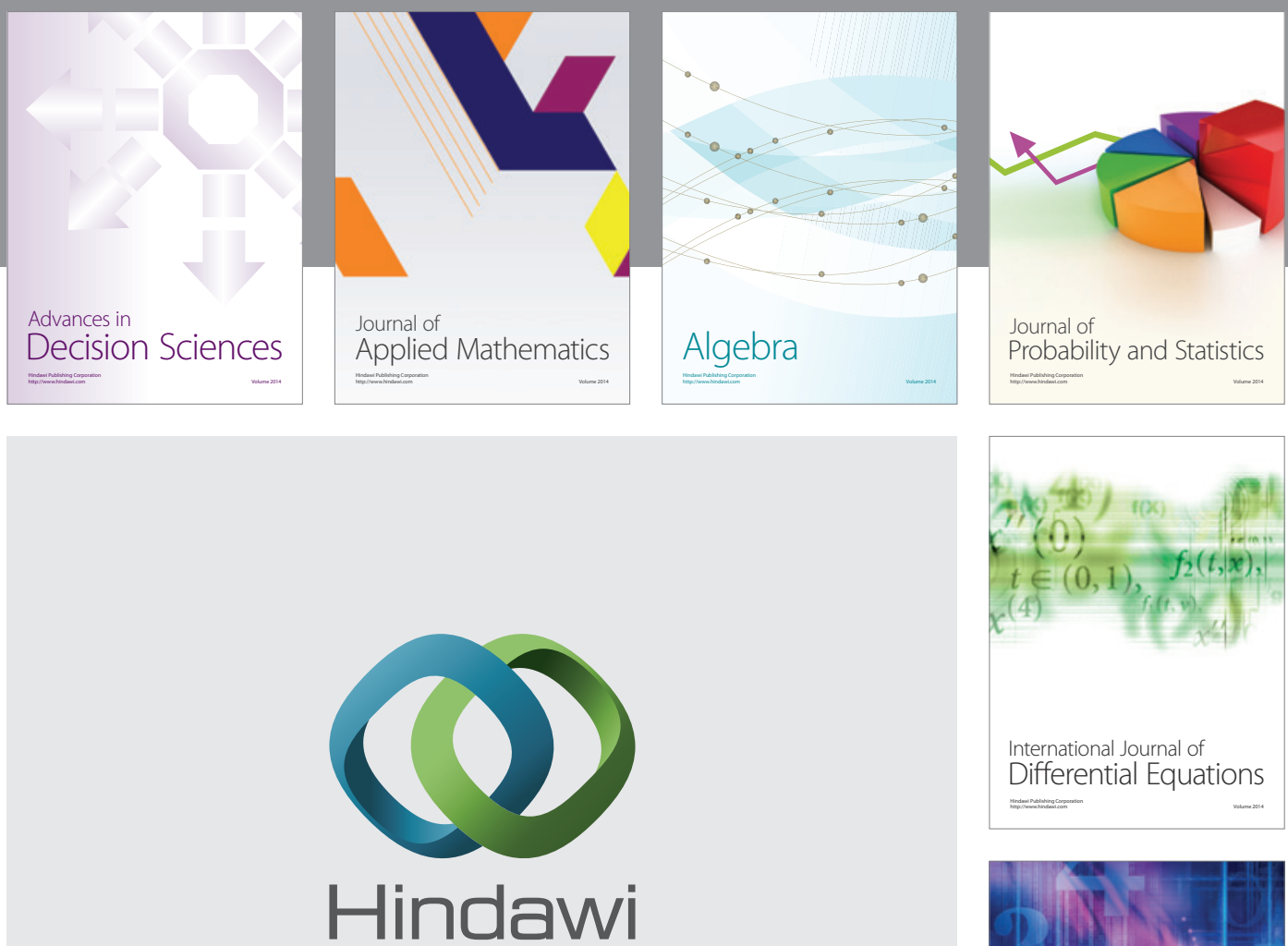

Submit your manuscripts at http://www.hindawi.com
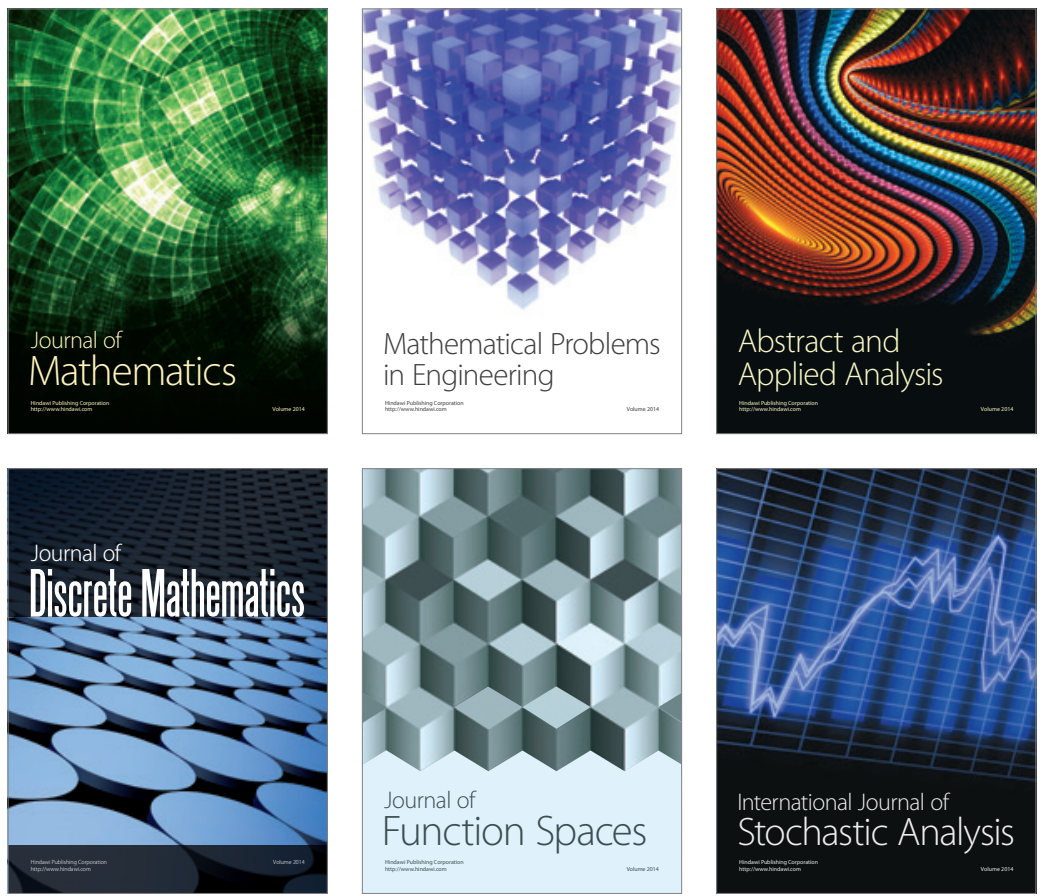

Journal of

Function Spaces

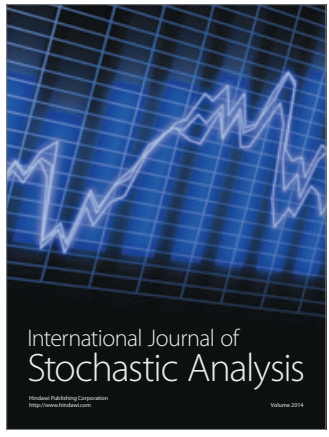

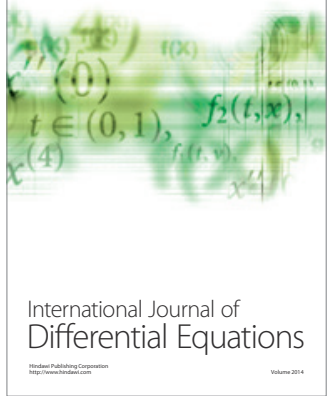
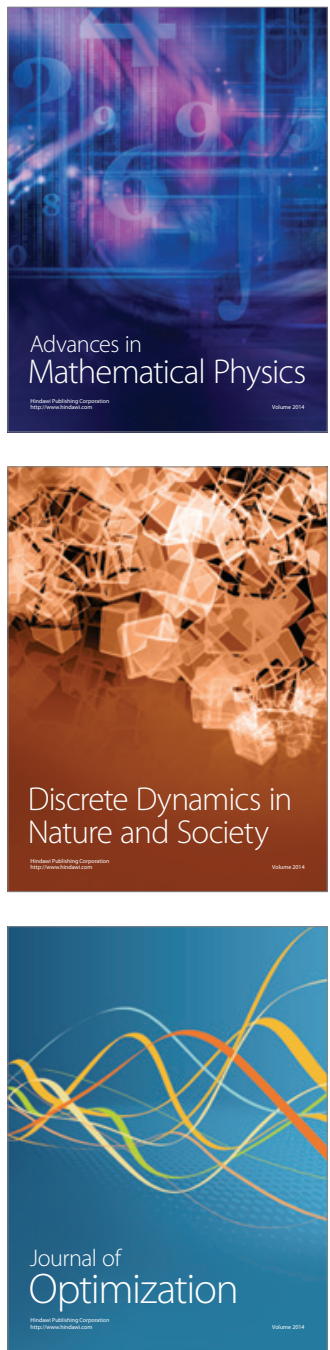